\title{
A Dual GTO Current-Source Converter Topology with Sinusoidal Inputs for High-Power Applications
}

\author{
Yuan Xiao, Bin Wu, Member, IEEE, Frank A. DeWinter, Senior Member, IEEE, and Reza Sotudeh, Member, IEEE
}

\begin{abstract}
A dual gate-turn-off thyristor current-source converter topology with sinusoidal inputs is proposed for high-power applications. The sinusoidal input current is realized by using pulsewidth modulation techniques to eliminate 11th and 13th harmonics and a transformer to cancel 5th, 7th, 17th, and 19th harmonics. Three switching patterns are proposed with a switching frequency of 360 or $420 \mathrm{~Hz}$. The combination of these switching patterns provides a full-range control over the dc output current. Resonant modes of the proposed system are identified, and the criterion for the line capacitor design is provided. Simulation and experimental results are given to verify the theoretical analysis.
\end{abstract}

Index Terms-AC motor drives, gate-turn-off (GTO) thyristor converters, high-power rectifiers, pulsewidth modulation techniques.

\section{INTRODUCTION}

$\mathbf{I}$ N HIGH-POWER (up to $10000 \mathrm{hp}$ ) ac motor drives using gate-turn-off (GTO) thyristor current-source inverter technology, SCR rectifiers are often used as front-end converters [1], [2]. The SCR rectifier has the features of simple structure, reliable operation, and bidirectional power flow. However, it injects harmonic currents into the power systems, and its power factor is poor under light-load conditions. A possible solution to these problems is to replace the SCR rectifier with a GTO pulsewidth modulation (PWM) current-source converter [3]. Fig. 1 shows a simplified circuit diagram of a GTO ac/dc current-source converter which can be used to replace an SCR rectifier in high-power induction motor drivers. Typically, the GTO devices are required to be connected in series in medium-voltage (4160-6900 V) applications.

For the design of a high-power GTO current-source converter, one of the most important issues is the switching frequency, which should be kept as low as possible to minimize GTO switching and snubber power loss. This requirement

Paper IPCSD 98-17, presented at the 1997 IEEE Applied Power Electronics Conference and Exposition, Atlanta, GA, February 23-27, and approved for publication in the IEEE TRANSACTIONS ON INDUSTRY APPLICATIONS by the Industrial Power Converter Committee of the IEEE Industry Applications Society. Manuscript released for publication March 3, 1998.

Y. Xiao and B. Wu are with the Department of Electrical and Computer Engineering, Ryerson Polytechnic University, Toronto, Ont., M5B 2K3 Canada.

F. A. DeWinter is with Rockwell Automation/Allen Bradley Canada Ltd., Cambridge, Ont., N1R 5X1 Canada.

R. Sotudeh is with the Department of Electronics and Computer Engineering, University of Teesside, Middlesbrough, Cleveland, TS1 3BA U.K.

Publisher Item Identifier S 0093-9994(98)05180-9.

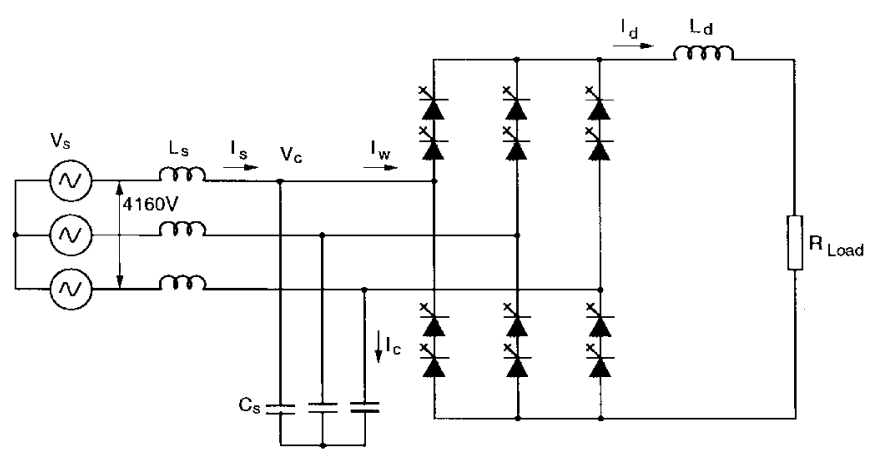

Fig. 1. Circuit diagram of a high-power GTO current-source converter.

is also imposed by the switching characteristics of high-power GTO devices [4], [5].

In order to minimize the switching frequency, while keeping the input current close to sinusoidal, a novel GTO currentsource converter topology, as shown in Fig. 2, is proposed. This topology is composed of two identical converters and an isolation transformer. The transformer is used to cancel certain harmonics produced by the converters. The other loworder harmonics that cannot be cancelled by the transformer are eliminated by PWM switching patterns. Compared with the single converter topology, the proposed dual converter has the following potential features.

- Sinusoidal input current-The transformer is used to cancel 5th, 7th, 17th, and 19th harmonic currents, while the PWM technique is employed to eliminate 11th and 13th harmonics. As a result, the input line current does not contain any harmonics of an order lower than 23rd. The other high-order harmonics can be easily filtered out by the line capacitor.

- Low switching frequency-As mentioned above, only 11th and 13th harmonics are required to be eliminated by the PWM pattern. Therefore, the lowest switching frequency for the proposed topology could be $360 \mathrm{~Hz}$. For the single converter to eliminate all the harmonics with the order lower than 23rd, the minimum switching frequency is $840 \mathrm{~Hz}$, which is too high to be implemented for high-power applications.

- Reliable operation for high-voltage applications-No GTO devices are connected in series in the proposed topology. The dynamic/steady-state voltage-sharing problem for the series devices in a single converter 


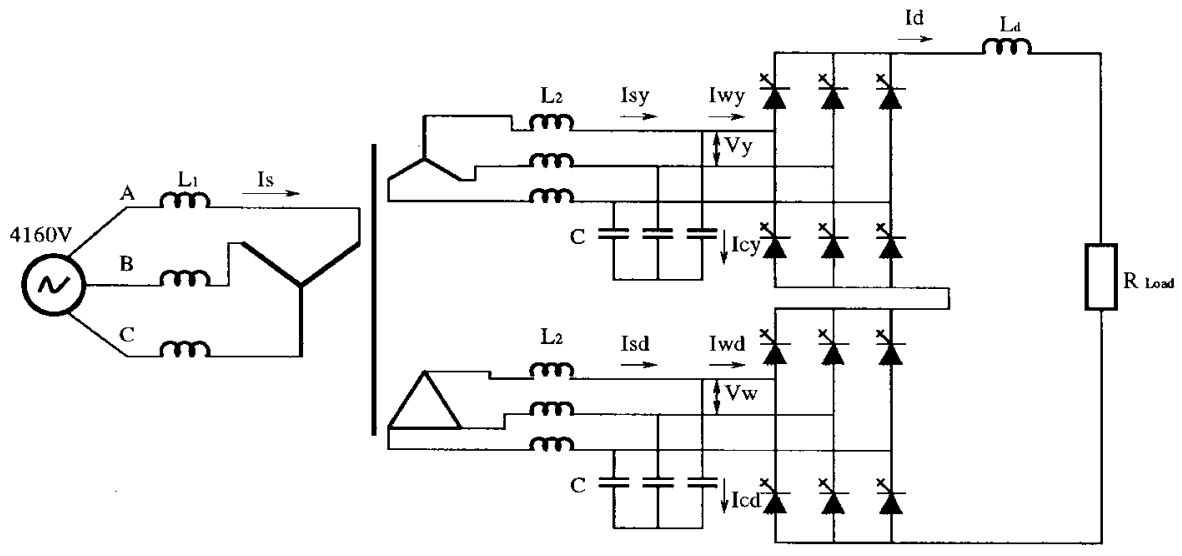

Fig. 2. A dual GTO PWM current-source converter configuration.

topology is completely avoided. The number of GTO devices for the dual converter topology remains the same as that for the single converter topology. For example, in a drive system with a supply voltage of $4160 \mathrm{~V}$, twelve $6000-\mathrm{V}$ GTO devices are required for both single and dual converter topologies. This concept can be easily used to develop a triple converter topology for higher voltage applications.

Compared with a single GTO converter topology, the proposed topology requires a transformer, which may not be considered as a disadvantage in terms of cost and converter size. For example, in retrofit or new applications where a standard (off-the-shelf) ac motor is used, an isolation transformer between the utility supply and front-end converter is indispensable to eliminate excessive line-to-ground and neutral-to-ground motor voltage generated by the currentsource drives [6]. The transformer used in the dual converter topology serves the same purpose in addition to the harmonic cancellation. Therefore, the proposed topology is particularly suitable for this type of application. Compared to the single converter with an isolation transformer, the cost increase of the proposed dual converter system is minimal, mainly because the number of GTO devices, snubbers, and gating boards is essentially the same for both topologies.

\section{HARMONIC CANCELlation}

It is assumed that two sets of the transformer secondary windings are connected with a $30^{\circ}$ phase shift. It can be proved that, regardless of the current waveforms in the secondary windings, the 5 th, 7 th, 17 th, 19th, 27th, and 29th harmonic currents in these windings will be cancelled and do not appear in the primary windings.

\section{SWitching PATTERnS}

The basic requirements for the design of switching patterns for the proposed topology are as follows:

- to eliminate 11th and 13th harmonics;

- to provide an adjustable dc current over a full range by adjusting modulation index;

- to minimize switching frequency.
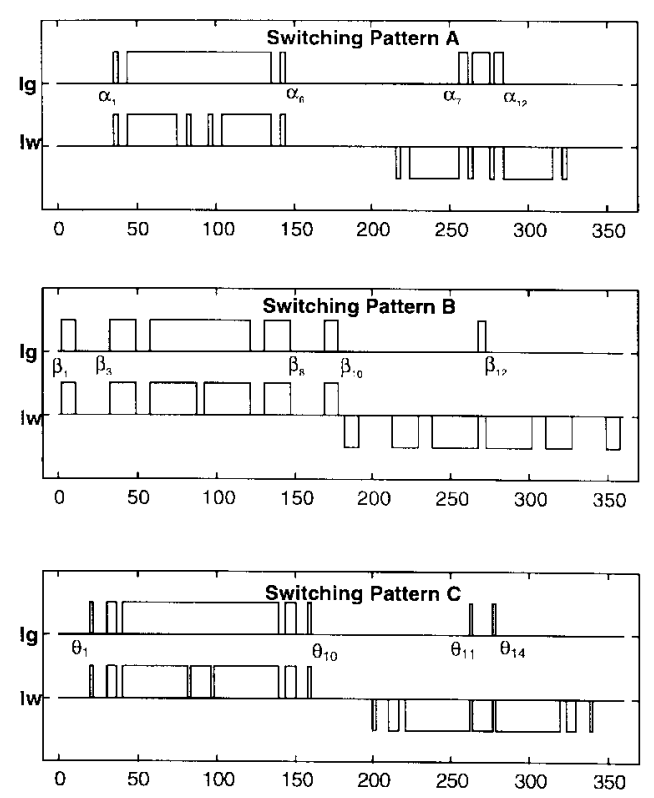

Fig. 3. Three proposed switching patterns. Pattern $A M_{d}=0.02 \sim 0.857$, $f_{s w}=360 \mathrm{~Hz}$. Pattern $B M_{d}=0.84 \sim 1.086, f_{s w}=360 \mathrm{~Hz}$. Pattern $C$ $M_{d}=0.84 \sim 1.086, f_{s w}=420 \mathrm{~Hz}$.

Besides these requirements, the switching pattern design must satisfy a constraint, that is, only one switching device in the upper legs of the converter and one in the lower legs can be turned on at any time to guarantee a continuous $\mathrm{dc}$ output current and a defined converter input current.

Fig. 3 shows three switching patterns developed for the dual converter topology. Patterns $A$ and $B$ have a switching frequency of $360 \mathrm{~Hz}$, which is the lowest possible frequency to satisfy the first two requirements, while Pattern $C$ has a switching frequency of $420 \mathrm{~Hz}$. The modulation index for the converter input current $I_{w}$ is defined as

$$
M_{d}=\frac{A_{1}}{I_{\mathrm{dc}}}
$$

where $A_{1}$ is the amplitude of fundamental component in $I_{w}$ and $I_{\mathrm{dc}}$ is the dc current of the converter.

Fig. 4 shows the converter input current waveform produced by Pattern $B$ for switching angle calculation. To satisfy the constraint imposed by the current source converter, the switch- 


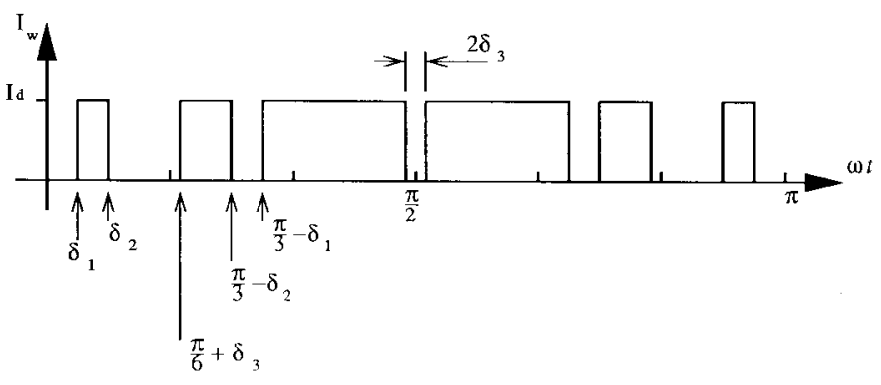

Fig. 4. Definition of independent variables.

ing angles are arranged such that there are only two switches conducting at any given time instant. As a result, only three switching angles, $\delta_{1}, \delta_{2}$, and $\delta_{3}$, in Fig. 4 are independent. Once these angles are determined, all other angles in a half cycle can be readily calculated. The converter current $I_{w}$ can be expressed by a Fourier series

$$
I_{w}(\omega t)=\sum_{n=1}^{\infty} A_{n} \sin (n \omega t)
$$

where $A_{n}$ is the amplitude of the $n$ th-order harmonic which can be calculated by

$$
\begin{aligned}
A_{n}= & \frac{4 I_{\mathrm{dc}}}{\pi}\left\{\cos \left(n \delta_{1}\right)-\cos \left(n \delta_{2}\right)\right. \\
& +\cos \left[n\left(\frac{\pi}{6}+\delta_{3}\right)\right]-\cos \left[n\left(\frac{\pi}{3}-\delta_{2}\right)\right] \\
& \left.+\cos \left[n\left(\frac{\pi}{3}-\delta_{1}\right)\right]-\cos \left[n\left(\frac{\pi}{2}-\delta_{3}\right)\right]\right\} .
\end{aligned}
$$

To eliminate 11th and 13th harmonics, two equations can be obtained by setting $A_{11}$ and $A_{13}$ to zero.

A third equation is required to obtain a desired modulation index. For a given $M_{d}$, the equation can be obtained by setting

$$
A_{1}-M_{d} I_{\mathrm{dc}}=0 .
$$

Thus, three independent variables $\delta_{1}, \delta_{2}$, and $\delta_{3}$ can be obtained by solving three nonlinear equations simultaneously.

Fig. 5 illustrates the calculated switching angles for Patterns $A$ and $B$. It can be observed that, with the increase of modulation index $M_{d}$, the switching angle $\alpha_{9}$ increases, while $\alpha_{10}$ decreases. When these two angles are merged at $M_{d}=0.857$, the corresponding pulsewidth becomes zero, at which the simultaneous equations given by (3) and (4) have no solution and the 11th and 13th harmonics are no longer eliminated. Therefore, Pattern A can be used with the modulation index lower than 0.857 .

When the modulation index $M_{d}$ decreases from its maximum value of 1.085 , the angle $\beta_{4}$ of Pattern $B$ approaches $\beta_{3}$ and, at the same time, the angle between $\beta_{7}$ and $\beta_{8}$ decreases. These angles are merged at $M_{d}=0.84$, which is the lowest modulation index for Pattern $B$. Fortunately, an overlap between Patterns $A$ and $B$ exists, which allows the converter to operate in a full modulation range. The converter can change its operating mode from Pattern A to Pattern $B$ at the modulation index of 0.85 , which is the mean value of the maximum modulation index of Pattern $A$ and the minimum modulation index of Pattern B. Pattern $C$ has the same range

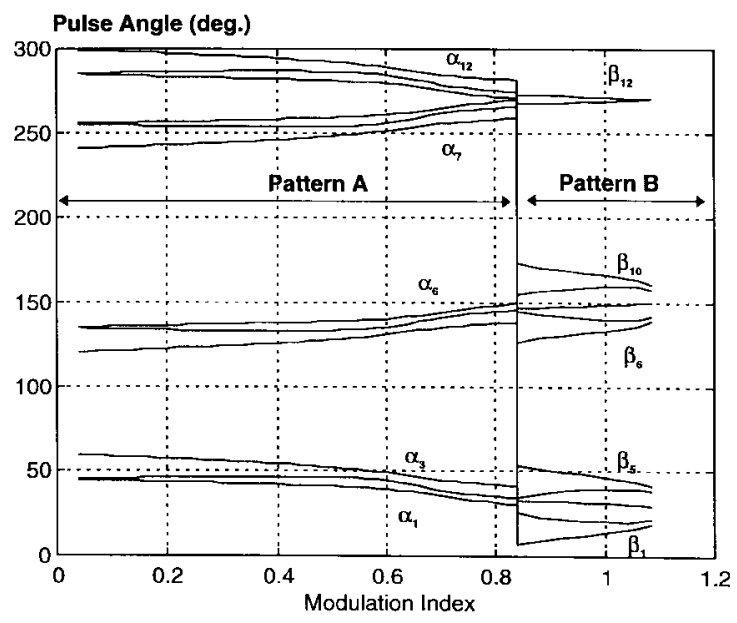

Fig. 5. Switching angle versus modulation index.

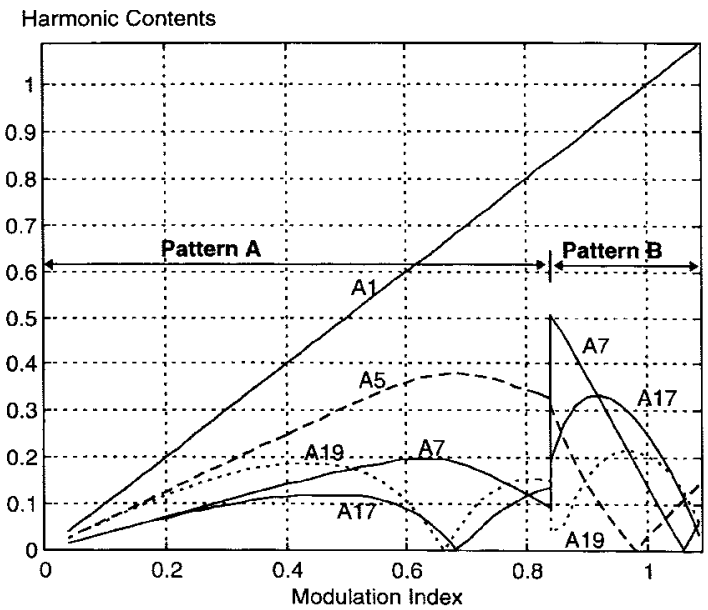

Fig. 6. Harmonic contents of combined PWM Patterns $A$ and $B$.

of modulation index as Pattern $B$ and, therefore, will not be discussed here.

Fig. 6 shows the harmonic contents in the converter input current generated by these two patterns. Although these switching patterns can satisfy all the requirements, the 7 th and 17th harmonic currents produced by Pattern $B$ are relatively high, which may increase energy loss in the transformer secondary winding. Fig. 7 illustrates the harmonic content associated with Patterns $A$ and $C$. Obviously, a better harmonic profile is achieved for Pattern $C$, at the expense of increased switching frequency. Furthermore, the magnitude of harmonic currents changes with the modulation index smoothly, especially during the transit between the two patterns. Therefore, for high-power converters where a switching frequency of 420 $\mathrm{Hz}$ can be implemented, the combination of Patterns $A$ and $C$ is recommended.

\section{Resonant Modes and Capacitor Design}

The filter capacitor and transformer inductances constitute the system resonant modes. Fig. 8 shows the equivalent circuit for resonant mode analysis. The system admittance seen by 


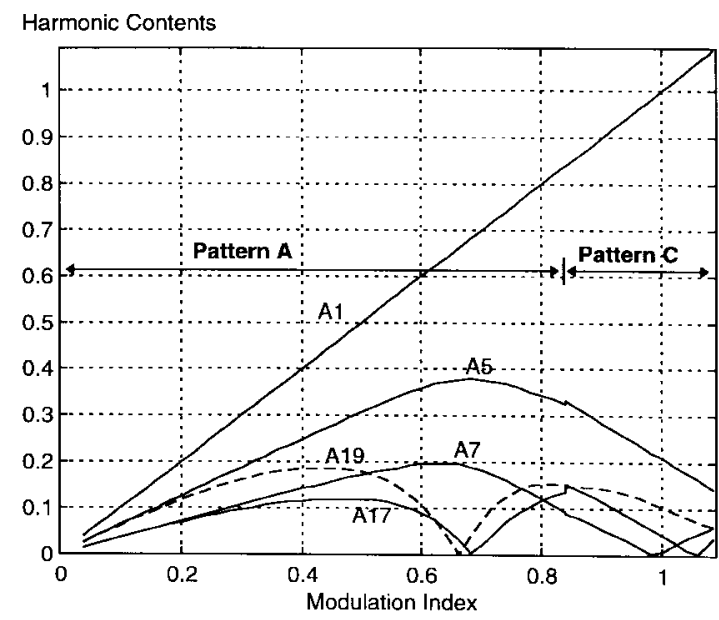

Fig. 7. Harmonic contents of combined PWM Patterns $A$ and $C$.

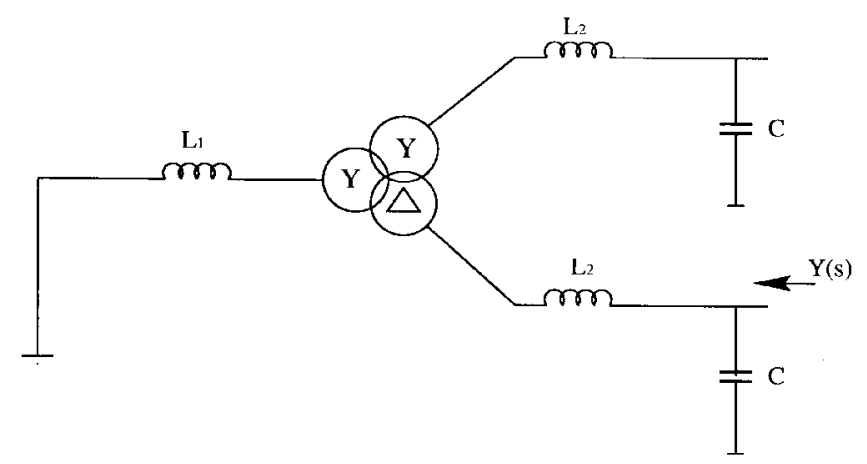

Fig. 8. Equivalent circuit for resonant mode analysis.

the converter can be expressed as

$$
Y(s)=\frac{1}{S L_{2}+\frac{1}{\frac{1}{S L_{1}}+\frac{S C}{S^{2} L_{2} C+1}}} .
$$

The zeros of the admittance $Y(s)$ represent the parallel resonant modes. The frequencies of these resonant modes can be calculated by

$$
\omega_{1}=\frac{1}{\sqrt{L_{2} C}}
$$

and

$$
\omega_{2}=\frac{1}{\sqrt{\left(2 L_{1}+L_{2}\right) C}} .
$$

The first resonant mode is associated with the transformer secondary leakage inductance only. This resonance may be excited by the harmonics in the converter input current $I_{w}$. Since the 11th and 13th harmonic currents in $I_{w}$ are eliminated, the frequency of this resonant mode may be set to

$$
\omega_{1}=11-13 \text { p.u. }
$$

Assuming the secondary leakage inductance $L_{2}$ is 0.05 per unit, the capacitor size can be determined by

$$
C=\frac{1}{\omega_{1}^{2} L_{2}}=0.12-0.17 \text { p.u. }
$$

This equation also indicates that the capacitor size could be reduced by increasing the transformer secondary leakage inductance, which can be achieved by transformer design. (a)

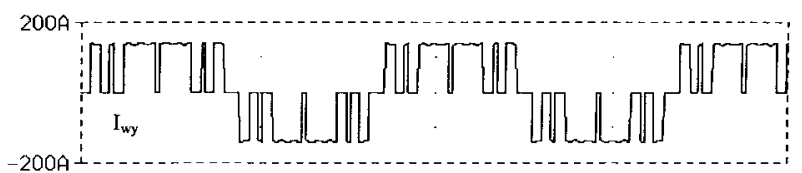

(b)

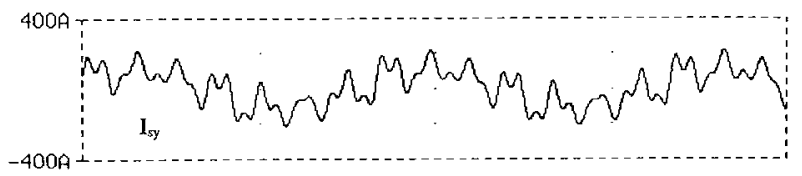

(c)

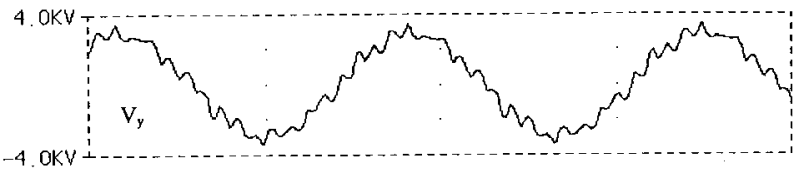

(d)

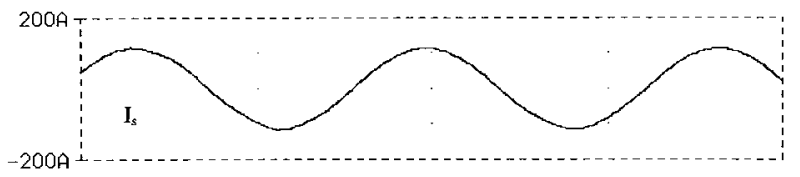

(e)

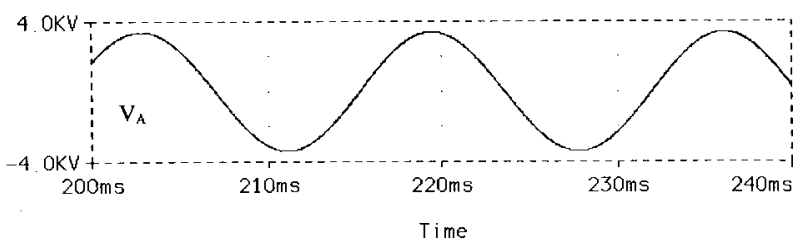

Fig. 9. Simulation results from a 4160-V 1-MVA converter system. Pattern $B$ at $M_{d}=0.9$ (the worst operating condition).

The transformer winding can be arranged in such a way that some of the primary leakage inductance can be moved to the secondary side without increasing the cost of the transformer.

The second resonant mode is dominated by the total inductance $L_{1}$ on the transformer primary side including the inductance of the utility supply. Assuming $L_{1}=0.15$ per unit, the resonant frequency is

$$
\omega_{2}=\frac{1}{\sqrt{\left(2 L_{1}+L_{2}\right) C}}=4.1-4.9 \mathrm{pu} .
$$

Since the current in the primary winding does not contain any low-order harmonics, this resonance will not be excited.

The resonant frequencies given in (6) and (7) are derived under the assumption that the equivalent $Y$ - and $\Delta$-connected secondary leakage inductances (refer to Fig. 8) have the same value. These inductances can be made equal during the transformer design process. However, in manufacturing, a few percentages of discrepancy may occur. In what follows, the effect of such a discrepancy on the resonant frequency is discussed. Assume that the $Y$ - and $\Delta$-connected secondary leakage inductances can be expressed as

$$
L_{Y}=L_{2}
$$

and

$$
L_{\Delta}=(1+K) L_{2}
$$

where $K$ represents the discrepancy in percent.

Following the same procedure discussed at the beginning of this section, the frequencies of the resonant modes can be 


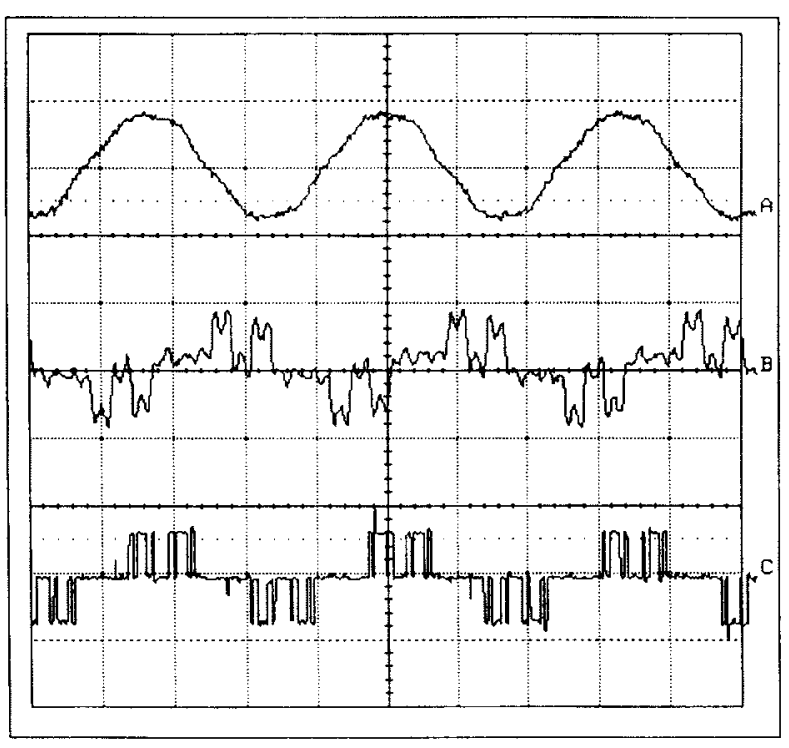

(a)

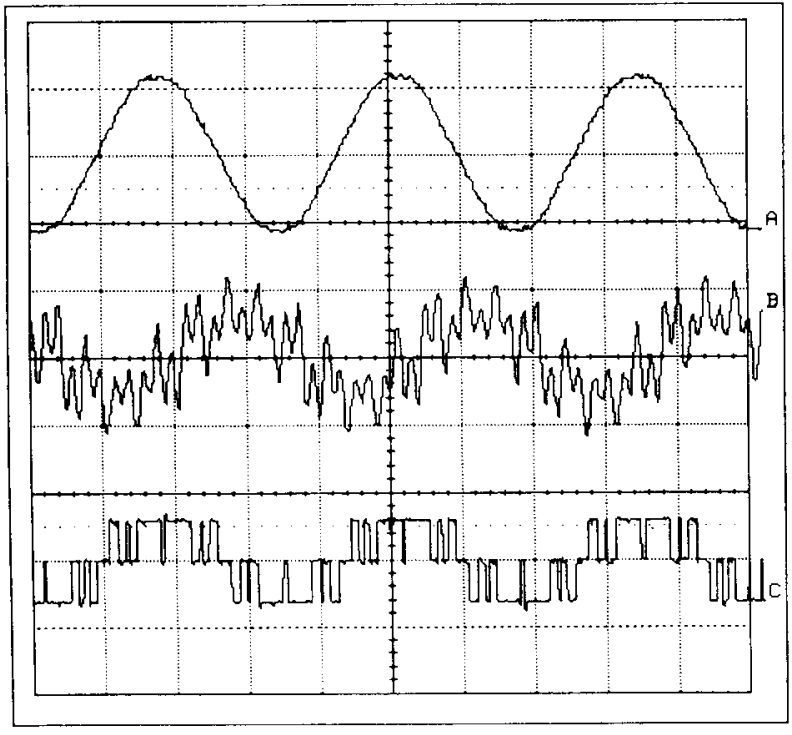

(b)

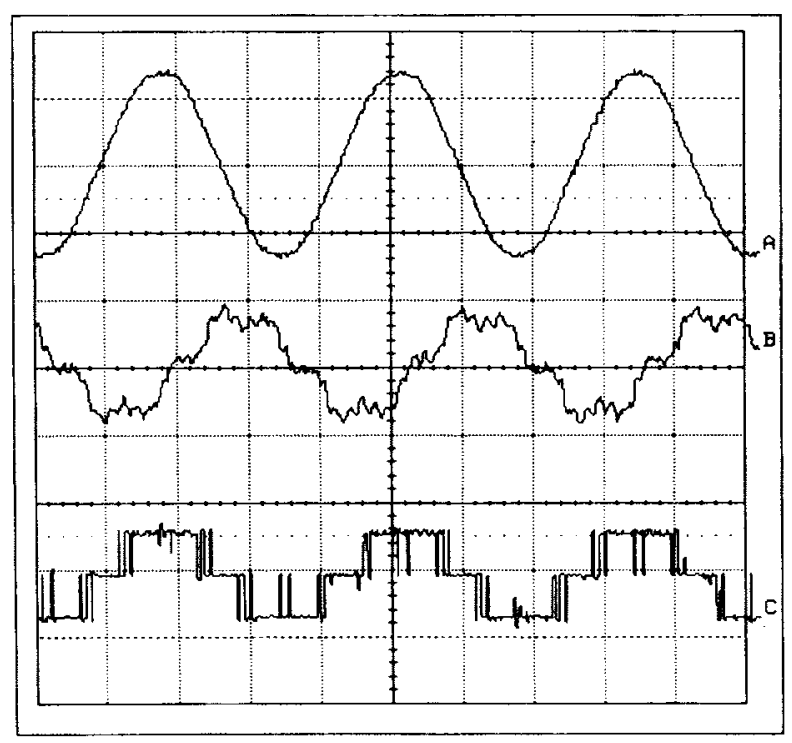

(c)

Fig. 10. Experimental results from a 208-V 20-kVA dual converter system. (a) Pattern $A$ at $M_{d}=0.5$. (b) Pattern $B$ at $M_{d}=0.9$ and (c) Pattern $C$ at $M_{d}=1.02$. Trace $A$ : line current $I_{s}, 50 \mathrm{~A} / \mathrm{div}, 5 \mathrm{~ms} / \mathrm{div}$. Trace $B$ : transformer secondary current $I_{s d}, 50 \mathrm{~A} / \mathrm{div}, 5 \mathrm{~ms} / \mathrm{div}$. Trace $C$ : converter input current $I_{w d}, 50 \mathrm{~A} / \mathrm{div}, 5 \mathrm{~ms} / \mathrm{div}$.

calculated by

$$
\begin{aligned}
\omega_{1}= & \frac{1}{\sqrt{C L_{2}}} \cdot \frac{1}{\sqrt{1+\frac{L_{1} K}{2 L_{1}+L_{2}(1+K)}}} \\
& \approx \frac{1}{\sqrt{C L_{2}}} \cdot \frac{1}{\sqrt{1+K / 2}}
\end{aligned}
$$

and

$$
\begin{aligned}
\omega_{2}= & \frac{1}{\sqrt{2 C\left(L_{1}+L_{2} \frac{1+K}{2+K}\right)}} \\
& \approx \frac{1}{\sqrt{C\left(2 L_{1}+L_{2}\right)}} \cdot \sqrt{\frac{1+K / 2}{1+K}} .
\end{aligned}
$$

If the secondary leakage inductances have a 5\% discrepancy, $\omega_{1}$ and $\omega_{2}$ will approximately change $1.2 \%$. Obviously, this change has little effect on the converter operation.

\section{InPUT POWER FACTOR CONTROL}

It is well known that a capacitor bank is required in currentsource converters to assist the commutation of switching devices. The use of the capacitor will make the input power factor leading. In the proposed converter system, a relatively small size capacitor can be used, even though the switching frequency is only 360 or $420 \mathrm{~Hz}$. This feature will facilitate the implementation of unity power factor operation. With a typical capacitor value of 0.15 per unit for each converter, the converter system will have a leading input power factor of 0.96 under rated load conditions. To achieve unity power 
TABLE I

Total Harmonic Distortion From Experimental Results

\begin{tabular}{c|c|c|c}
\hline Switching Pattern & $\begin{array}{c}\text { Switching } \\
\text { Frequency (Hz) }\end{array}$ & Modulation Index & THD: $I_{s}$ \\
\hline A & 360 & 0.5 & $5.4 \%$ \\
\hline B & 360 & 0.9 & $1.8 \%$ \\
\hline C & 420 & 1.02 & $1.4 \%$ \\
\hline
\end{tabular}

factor, a phase-shift control can be integrated with modulation index control [7]. A small phase shift between the converter input voltage and modulated current will easily make the input power factor unity. A comprehensive analysis of the input power factor control for high-power GTO current-source converters is presented in [8].

\section{SimUlation AND EXPEIRMENTAL RESUltS}

Fig. 9 shows a set of the simulation results. The converter system is rated at $4160 \mathrm{~V}$ (line-to-line), $60 \mathrm{~Hz}$, and $1000 \mathrm{kVA}$. The parameters used in the simulation are $L_{1}=0.15, L_{2}=$ 0.05 , and $C=0.13$, all in per unit. Switching Pattern $B$ is selected with the modulation index set at 0.9 , at which both 7 th and 17th harmonics have a large magnitude (the worst operating condition). The waveforms of converter input current $L_{w y}$, transformer secondary current $I_{s y}$, and secondary line-to-line voltage $V_{y}$ are shown in Fig. 9(a)-(c), respectively. Although the secondary current $I_{s y}$ contains harmonics, these harmonics can be cancelled by the $\Delta-Y$-connected transformer. Therefore, the line current $I_{s}$ on the primary side is sinusoidal. The unity power factor is obtained by introducing a small delay angle between the converter input current and voltage.

The experimental results are obtained from a laboratory GTO dual current-source converter system. The control of the laboratory unit, including PWM gate pulse generators, proportional integral (PI) controllers, and a unity power factor controller, is implemented by a TMS320C31-based digital signal processor (DSP) board. All three switching patterns proposed in this paper are included in the PWM generator. The converter system is rated at $208 \mathrm{~V}, 20 \mathrm{kVA}$, and 60 $\mathrm{Hz}$ with $L_{1}=0.026, L_{2}=0.051$, and $C=0.15$ per unit. The waveforms of converter input current $I_{w y}$, transformer secondary current $I_{s y}$, and line input current $I_{s}$ when the system is operated at $M_{d}=0.5,0.9$, and 1.02 with Switching Patterns $A, B$, and $C$ are shown in Fig. 10. As shown in Table I, the measured total harmonic distortion (THD) of the input line current is $5.4,1.8$, and $1.4 \%$, respectively.

To investigate possible resonances which may be caused by the resonant modes during transient, a step command is applied to the converter system. Fig. 11 shows one set of such experiments. The dc current $I_{d}$ is increased from zero to $32 \mathrm{~A}$ in $40 \mathrm{~ms}$. The transformer primary line current $I_{s}$, secondary line current $I_{s d}$, and converter input current $I_{w d}$ do not exhibit any resonant phenomenon during transient. Many experiments were performed under various loading conditions with a step increase or step decrease command. No resonant phenomena were ever observed during the experiments.

\section{CONCLUSIONS}

A dual GTO current-source converter topology with sinusoidal inputs has been proposed for high-power appli-

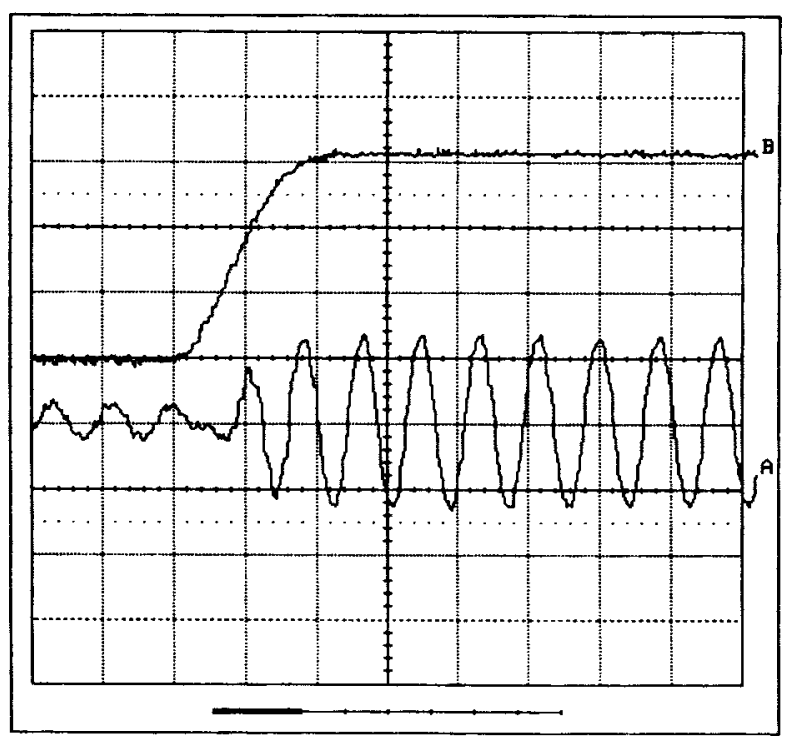

(a)

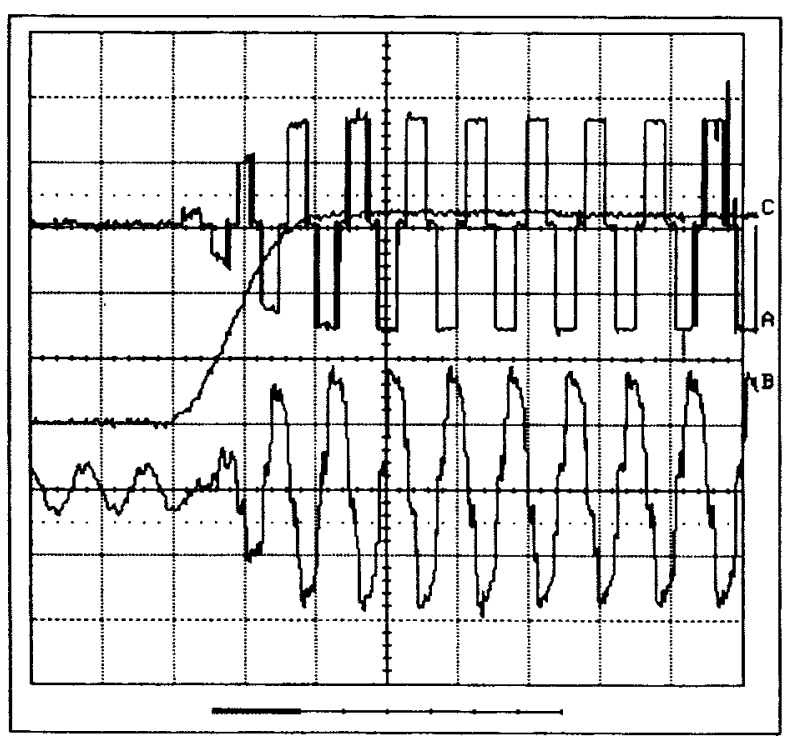

(b)

Fig. 11. Step response of laboratory dual converter system for the investigation of resonant modes, Pattern $B$ at $M_{d}=1.075$. Trace $A$ : converter input current $I_{w d}, 50 \mathrm{~A} / \mathrm{div}, 20 \mathrm{~ms} / \mathrm{div}$. Trace $B$ : transformer secondary current $I_{s d}$, $50 \mathrm{~A} / \mathrm{div}, 20 \mathrm{~ms} / \mathrm{div}$. Trace $C$ : dc current $I_{\mathrm{dc}}, 10 \mathrm{~A} / \mathrm{div}, 20 \mathrm{~ms} / \mathrm{div}$.

cations. The sinusoidal input current is realized by using PWM techniques to eliminate 11th and 13th harmonics and a transformer which is connected to two identical currentsource converters. Three switching patterns are developed with switching frequencies of 360 and $420 \mathrm{~Hz}$. The combination of these switching patterns provides a full-range control over the dc output current. Resonant modes of the proposed system are identified, and the criterion for the line capacitor design is provided. A unity power factor control scheme for the proposed topology is briefly discussed. A 20-kVA dual current-source converter system has been constructed to verify the theoretical analysis. The proposed topology is particular suitable for high-power applications, due to its low switching frequency, sinusoidal inputs, and unity power factor operation. 


\section{REFERENCES}

[1] P. M. Espelage and J. M. Nowak, "Symmetrical GTO current source inverter for wide speed range control of 2300-4160 volt, 350 to 7000 hp, induction motors," in Conf. Rec. IEEE-IAS Annu. Meeting, 1988, pp. 302-307.

[2] B. Wu, S. A. Dewan, and G. R. Slemin, "PWM-CSI inverter for induction motor drives," IEEE Trans. Ind. Applicat., vol. 28, pp. 64-71, Jan./Feb. 1992.

[3] M. Iwahori and K. Kousaka, "Three-phase current source rectifier adopting new PWM control techniques," in Conf. Rec. IEEE-IAS Annu. Meeting, 1989, pp. 855-860.

[4] H. R. Karshenas, H. A. Kojori, and S. B. Dewan, "Generalized techniques of selective harmonic elimination in current source inverters/converters," IEEE Trans. Power Electron., vol. 10, pp. 566-573, Sept. 1995.

[5] Y. Xiao, B. Wu, F. DeWinter, and R. Sotudeh, "High power GTO ac/dc current source converter with minimum switching frequency and maximum power factor," in Proc. CCECE, 1996, pp. 331-334.

[6] B. Wu and F. DeWinter, "Voltage stress on induction motors in medium voltage (2300-6900 V) PWM GTO CSI Drives," in Conf. Rec. IEEE PESC'95, 1995, pp. 1128-1132.

[7] J. H. Choi, H. A. Kojori, and S. B. Dwan, "High power GTO-CSC based power supply utilizing SHE-PWM and operating at unity power factor," in Proc. CCECE, 1993.

[8] Y. Xiao, B. Wu, S. Rizzo, and R. Sotudeh, "A novel power factor control scheme for high power GTO current source converter," in Conf. Rec. IEEE-IAS Аnnu. Meeting, 1996, pp. 865-869.

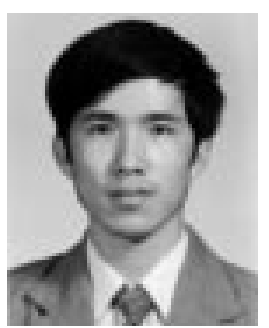

Yuan Xiao received the B.Sc and M.Eng. degrees in electrical engineering from Xi'an Jiaotong University, Xi'an, China, in 1982 and 1985, respectively, and the M.A.Sc. degree from the University of Toronto, Toronto, Ont., Canada, in 1993. He is currently working towards the Ph.D. degree in electrical engineering at Ryerson Polytechnic University, Toronto, Ont., Canada, under a joint program with the University of Teesside, Middlesbrough, Cleveland, U.K.

Since 1996, he has been a part-time employee with Rockwell Automation/Allen Bradley Canada Ltd., Cambridge, Ont., Canada, where he is working on high-power converter systems. His areas of interest include high-power converter design and power system modeling and analysis.

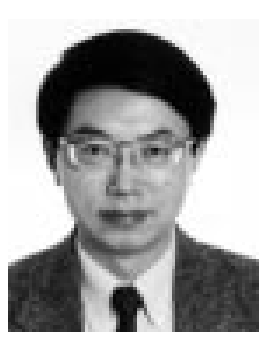

Bin Wu (S'89-M'91) received the M.A.Sc. and $\mathrm{Ph} . \mathrm{D}$. degrees in electrical engineering from the University of Toronto, Toronto, Ont., Canada, in 1989 and 1993 , respectively.

Following employment with Rockwell Automation/Allen Bradley Canada Ltd. as a Senior Development Engineer, he joined Ryerson Polytechnic University, Toronto, Ont., Canada, where he is currently an Associate Professor. His research interests include power converter topologies, motor drives, computer simulation, and DSP applications in power engineering.

Dr. Wu was awarded the Gold Medal of the Governor General of Canada in 1990. He is a Registered Professional Engineer in the Province of Ontario, Canada.

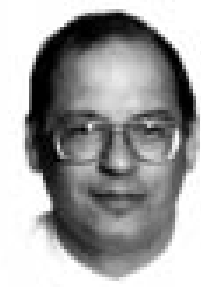

Frank A. DeWinter (M'82-SM'90) received the Electrician Certificate from Northern Alberta Institute of Technology, Alta., Canada, and the B.Sc. degree in electrical engineering from the University of Alberta, Edmonton, Alta., Canada, in 1976 and 1980, respectively.

From 1980 to early 1990, he was with Colt Engineering Corporation as the Lead Electrical Engineer on projects for refineries, pipeline pump stations, petrochemical plants, and tor sand handling. Currently, he is the Director of Research and Development, Medium-Voltage Products, Rockwell Automation/Allen Bradley Canada Ltd., Cambridge, Ont., Canada, where he manages the research and development of medium-voltage products, including mediumvoltage drives, soft starters, and electromechanical starters. He has published ten previous papers on the subject of VFD and harmonics.

Mr. DeWinter is a Registered Professional Engineer in the Province of Ontario, Canada.

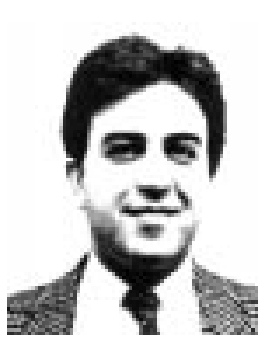

Reza Sotudeh (M'91) received the B.Sc. (Hons.) and Ph.D. degrees in electronics and computer engineering from the University of Sunderland, U.K., in 1981 and 1984, respectively.

He was a Group Leader with Microtechnology Ltd. from 1984 to 1986 and a Lecturer with the University of Sunderland from 1986 to 1987 . He became a Senior Lecturer and a Reader at the University of Teesside, Middlesbrough, Cleveland, U.K., in 1987 and 1990, respectively. He served as the Head of the Division of Electronics and Computer Engineering, University of Teesside, from 1991 to $1997 . \mathrm{He}$ became a Professor and the Head of Electrical and Electronics Engineering in 1995 and 1998, respectively. He is currently a SONY Professor of Computer Engineering, University of Teesside, and an Adjunct Professor to the Department of Electrical and Computer Engineering, Ryerson Polytechnic University, Toronto, Ont., Canada. His research interests are in the areas of application of microelectronics to power engineering problems, computer architecture, high-speed computer buses, and media processing.

Dr. Sotudeh is a Chartered Engineer in the U.K., a member of the Institution of Electrical Engineers (U.K.), and a Fellow of the Royal Society for Arts, Commerce, and Manufactures. 